\title{
$\alpha$-linolenic omega-3 fatty acid for stroke protection: from brain preconditioning paradigm to nutrition
}

\author{
Nicolas BLONDEAU \\ Université de Nice Sophia Antipolis, \\ 28 Avenue de Valrose, \\ 06103 Nice Cedex 2, \\ France; \\ Institut de Pharmacologie Moléculaire \\ et Cellulaire, \\ CNRS, \\ UMR 6097, \\ 660 Route des Lucioles, \\ 06560 Valbonne, \\ France \\ <blondeau@ipmc.cnrs.fr>
}

\begin{abstract}
Stroke is the third leading cause of death, due to its high incidence, the severity of the insult, and lack of treatment options. The only therapeutic is restoration of cerebral blood flow achieved by recombinant tissue plasminogen activator treatment, but only approximately 5\% of patients receive it. In addition, therapeutics aimed at achieving neuroprotection by blocking the ischemic cascade, as identified in numerous preclinical studies, failed in clinical trials. This failure in translation from experimental models to clinical trials led to a re-evaluation of properties which would constitute the "best-in class" therapeutics to be used against stroke. Given that neuroprotection appears ineffective per se, an emerging direction is to identify therapies, probably combinatorial in nature, which protect the whole neurovascular unit and target timedependent neurotoxic mechanisms. Molecules that activate complex cellular signaling cascades that render the brain resistant to subsequent ischemia, known as preconditioners, offer a novel perspective in stroke protection. Preconditioning elicits complex endogenous neuroprotective responses that act by pleiotropic mechanisms to block death pathways, promote survival pathways and increase resistance. In addition to chemical preconditioners, natural/endogenous compounds such as adenosine, glutamate, lysophospholipids, and omega-3 polyunsaturated fatty acids have been demonstrated to be excellent preconditioners. Consequently, a major new concept in preconditioning to combat stroke is introduced, which is preconditioning achieved through supplementation of an essential item in diet or as a nutraceutical. Several epidemiologic studies suggested a beneficial effect of a seafood/omega-3-enriched diet in cerebral diseases, but the omega-3-induced protective mechanisms are still poorly identified. This review highlights how $\alpha$-linolenic acid $(A L A)$, the omega-3 polyunsaturated fatty acid precursor, protects the brain from in vivo and in vitro models of stroke, thus potentially fulfilling the goal of identifying the "best-in class" therapeutics against stroke. Also described is the surprising pleiotric nature of ALA in protecting neurons, vasodilating brain arteries and stimulating neuroplasticity. Importantly, feasibility of delivery has been demonstrated, since ALA supplementation can be achieved through modification of the daily diet, for which prevention of stroke-induced mortality and cerebral damage has been confirmed. Therefore evaluating ALA as an interesting preconditioner against stroke represents a novel and extremely relevant concept in the context of nutraceutical and functional food development.
\end{abstract}

Key words: nutraceutical, omega-3 polyunsaturated fatty acids, stroke, brain preconditioning and tolerance, prevention, rapeseed oil

150,000 French and 780,000 Americans. On average in the developed countries, stroke strikes once every 40 seconds and causes death every 4 minutes, with an estimated $25 \%$ death rate within the first week, and $50 \%$ of the patient population within 5 years after the brain attack. Among survivors, work capacity is compromised in $70 \%$ of victims, among which $30 \%$ need assistance with self-care. Stroke causes functional control impairment, paralysis, speech and sensory problems, memory and reasoning deficits, often leading to

death, annually afflicting approximately

To cite this article: Blondeau N. $\alpha$-linolenic omega- 3 fatty acid for stroke protection: from brain preconditioning paradigm to nutrition. OCL 2011; 18(5): 271-278. doi : 10.1684/ocl.2011.0389 
long-term disability, dementia and poststroke depression. Hence stroke is a global burden that affects all racial or ethnic groups indiscriminately. Its social and psychological costs are substantial in developed nations, as well as its economic cost, which have been estimated to be greater than 70 billion dollars in 2010 in the USA (Lloyd-Jones et al., 2010, Rosamond et al., 2008).

In eighty five percent of stroke cases, the neurovascular event causing the stroke is ischemic, meaning that blood flow to a part of the brain is disrupted due to occlusion of a blood vessel. Occlusion deprives the brain of nutrients and oxygen, eliciting a complex interplay of multiple cellular signaling pathways that damages the neurovascular unit (neuronal, glial and endothelial cells (ladecola, 2004)) within the affected territory. Within the core of the ischemic territory, blood flow is most severely restricted, and excitotoxic and necrotic cell death occurs within minutes. In the periphery of the core, termed the ischemic penumbra, reduced collateral blood flow buffers the full effects of the stroke. In this area, primarily apoptosis leads to a slower rate of cell death (Endres and Dirnagl, 2002).The only therapeutic available is recombinant tissue plasminogen activator, which restores cerebral blood flow by disrupting the blood clot causing vessel occlusion. Unfortunately, due to its restricted application, thrombolysis it is only perform in approximately $5 \%$ of the stroke population. Other therapeutics aimed at more directly blocking the cellular ischemic cascade, as identified in numerous preclinical studies, failed in clinical trials. In 2007 O'Collins reviewed 1026 neuroprotective treatments identified for acute stroke between 1957 and 2003. Of the 114 treatments which were tested (and failed) in clinical trials, no drug perform any better against all other drugs when tested against focal models of cerebral ischemia. In addition, these drugs displayed poor adherence to the series of criteria determined to identify neuroprotective agents with the best chance of success in clinical trials by the Stroke Therapy Academic Industry Roundtable (STAIR). This poor translation from experimental models to clinical trials raised the question of whether the best drugs were being chosen to proceed to clinical trial, leading to consideration of new ideas and recommendations for the development of strategies in combating stroke.

Regarding translation, much has been written about timing and dosing, failure to incorporate preclinical information into clinical trials, as well as acknowledgement that animal data may not adequately support translation of a neuroprotective drug to clinical (Jonas et al., 1999). Although animal models may not faithfully reproduce all multifactorial aspects of strokes in humans, animal models are representative of important clinically relevant pathophysiological features of human stroke. Therefore, the "best in class" drug should be tested and be efficient in several experimental mode of stroke, as well as in species which include primates. A second recommendation is to redefine the view of the pathology as a cerebrovascular and not exclusively as a neuronal disease. Nowadays, considering only neurobiological aspects of stroke defies increasing recognition that stroke triggers both cerebral endothelium dysfunction leading to CBF alteration, and neuronal, astroglial and oligodendroglial homeostasis disruption, as well as and microglial activation. Therefore the research and medical community had now moved towards a more integrative view of stroke taking in consideration the protection of all cell types within the entire neurovascular unit (fundamentally comprising endothelium, astrocyte and neuron). In order to be considered as a good candidate for a clinical trial, stroke treatment must have clear and demonstrable efficacy on all the neurovascular unit cells or at least must be carefully considered in terms of its actions on all cell types and neurovascular functions. Thus, an experimental drug should be better tested for safety and efficacy not only in neurons but also on cerebral endothelial cells, astrocytes, oligodendrocytes, microglia and so on (Lo and Rosenberg, 2009). A large body of basic research over the past two decades shows that ischemic stroke causes brain damage by multiple pathways, implicating mechanisms like excitotoxicity, oxidative stress and programmed cell death. The failure of stroke trials which targeted one or only a few of these pathways suggests that targeting a single element of a single pathway may not yield sufficient neuroprotection. Consequently, an emerging view is that combination or "multi " therapy, or discovering drugs exhibiting multimodal actions at multiple cell types, is required to address the multifactorial nature of stroke. Therefore, molecules that provoke multiple protective and regenerative mechanisms should be particularly attractive therapeutic agents (Lo, 2008; Minnerup and Schabitz, 2009; Zaleska, et al., 2009). A pertinent and effective experimental approach to identify such candidates would be to better understand how the brain protects itself against ischemia, through the study of brain preconditioning.

\section{Brain preconditioning is an innovative approach in discovery of novel cerebroprotective strategies against ischemic stroke}

This cellular and adaptative biological process conferring resistance to ischemia is called preconditioning. Particularly within the last two decades, preconditioning has attracted much interest within the clinical and basic neuroscientist communities, primarily due to promotion of protection and regeneration against stroke through direct and/or indirect mechanisms, involving multiple cell types. Therefore preconditioning is considered pleiotropic in nature. Ischemic preconditioning of the brain, heart and other organs refers to an endogenous protective process that is induced by a sublethal ischemia and which increases the tissue tolerance to a subsequent, normally lethal ischemia. Since its original description in the brain by Kitagawa in 1990, (Kitagawa et al., 1990), non-ischemic peconditioners including various sublethal insults like epilepsy, endotoxins, anoxia, hyperthermia and spreading depression were shown to also induce delayed tolerance to normally lethal forms of themselves, and additionally tolerance to ischemia, also named "cross-tolerance" (Plamondon et al., 1999; Tauskela and Blondeau, 2009).

A concern that potential clinical applications of brain preconditioning may be very limited due to the requirement of bringing neurons to the "brink of death" during preconditioning, has been circumvented by discoveries that preconditioning and its tolerance to 
ischemia may be induced or mimicked pharmacologically by chemicals like adenosine and $\mathrm{K}_{\mathrm{ATP}}$ channel agonists (Blondeau et al., 2000). Several other compounds including 3-nitropropionic acid and the glutamate receptor agonist, N-methyl-D-aspartate (NMDA) have been investigated in both in vivo and in vitro models of preconditioning (Tauskela and Blondeau, 2009). Moreover, retrospective case-control studies showed a clinical correlate of the experimental preconditioning paradigm, suggesting that patients with a history of transient ischemic attack (TIA) exhibit a decreased morbidity after stroke (O'Duffy et al., 2007; Weih et al., 1999), strongly suggesting that preconditioning is a pertinent and effective experimental concept in the human brain. Finally, with the recent demonstration that preconditioning can also be induced by a variety of natural product like polyunsaturated fatty acid and lysophospholipids, preconditioning studies provide an innovative approach for the discovery of novel cerebroprotective strategies (Blondeau et al., 2002a; Blondeau et al., 2002b).

Preconditioning induces two different temporal windows of protection against ischemia: the first window known as "rapid preconditioning" occurs within minutes after the stimulus used for induction of preconditioning and only lasts for around $1 \mathrm{~h}$, whereas the delayed window produces a more robust state of protection that usually develops $24 \mathrm{~h}$ after preconditioning induction, peaks at 72 hours fades 7 days later. In general, a two- to four-day interval between preconditioning and lethal ischemia provides the greatest protection, at least in the rodent brain. Increasing this interval generally reduces benefits, although in some studies a delay of up to two weeks could still result in some ischemic tolerance. Longer stimulation with low stimulation by non-ischemic preconditioners, while still not inducing toxicity, may generate more robust ischemic tolerance. All these facts, the molecular sensors, transducers and effectors of preconditioning have been already elegantly described in high-impact reviews (Kirino 2002; Dirnagl et al., 2003; Gidday, 2006; Obrenovitch, 2008). These reviews also underline key conceptual differences between preconditioning and neuroprotection strategy, notably the time frame and mechanism of protection. Preconditioning activates endogenous pro-survival responses, including genetic remodeling, to suppress the toxic signaling effects, whereas drug therapies are mostly designed to directly inhibit toxic signaling pathways occurring post-stroke. Thus preconditioning requires a certain delay in order to attain the subsequent maximal protection, so it is often inadequately perceived as a preventive strategy.

\section{The preventive strategies in stroke are aimed to decrease stroke incidence}

The poor translation from experimental models to clinical trials has led to adoption of additional strategies in combating stroke, most notably drawing attention to the importance of prevention. The prevention or/and treatment of the risk factors has emerged as a priority to reduce the occurrence of stroke. Some risk factors cannot be modifiable, such as a family history of cerebrovascular diseases, aging, male sex, and Hispanic or Black race (Allen and Bayraktutan, 2008). But the other risk factors including cardiovascular complications (atrial fibrillation, valvular heart disease, ischemic cardiomyopathy and carotid stenosis for example), hypertension, diabetes, hypercholesterolemia, cigarette smoking, increased inflammatory markers, dyslipidemia, and obesity may be addressed by life-style changes and pharmacologicals, to prevent or minimize the possibility of having a stroke. Modifiable risk factors often coexist and have been estimated to account for $60 \%$ $80 \%$ of stroke incidence in the general population (Allen and Bayraktutan, 2008; Moskowitz et al., 2010), and are often associated with improper nutrition causing imbalance in essential vitamins and nutriments. Many clinical and epidemiologic studies have shown that deficiency in vitamins, nutriments, and essential omega-3 polyunsaturated fatty acids may be risk factors of stroke per se. Low levels of fruits and vegetables in diet or improper omega- 3 intake, both in the form of $\alpha$-Linolenic Acid (ALA) and the Long Chain derivatives (LC-n-3), EicosaPentaenoic-Acid (EPA) and Docosa-Hexaenoic-Acid (DHA), are a risk factor for cardiovascular and cerebral diseases, including coronary heart disease and stroke (Dauchet et al., 2005; de Goede et al., 2011; Riediger et al., 2009; Simopoulos, 2008).

Importantly, besides reducing the risk of stroke, these factors may exhibit a protective role against stroke-induced damage, a field of study of potentially major relevance but inadequately addressed. Several epidemiologic studies in cerebral diseases identified beneficial effects of diets rich in omega- 3 poly-unsaturated fatty acids (PUFAs) by consumption of seafood (rich in LC-n-3: EPA and DHA) and/or vegetable oils rich in precursor (ALA), suggesting that omega-3 PUFAs may have a neuroprotective role. This notion received mechanistic support with the discovery that omega-3 activates the neuronal potassium TREK-1 channel, leading to neuronal membrane hyperpolarization. Hyperpolarization of synaptic terminals decreases glutamate release while blocking NMDA receptor activation at the post-synaptic level, a protective strategy with proven efficiency in animal model of stroke (Fink et al., 1998; Lauritzen et al., 2000). Besides providing acute neuroprotection, our laboratories evaluate the possibly that omega3 FA may also act as a preconditioner against ischemia. By fulfilling multiple requirements of prevention, acute protection and preconditioning, acting at multiple cell types, omega-3 FA may indeed be recognized as a "best-in class" therapeutic against stroke.

\section{Omega-3 $\alpha$-linolenic acid as an acute treatment in experimental model of cerebral ischemia and stroke}

During the acute phase of ischemic injury, glutamate excitotoxicity and hyperactivation of its receptors are the major destructive mechanisms within the core and surrounding penumbra. Chronologically, glutamate releasedriven neuronal death occurs within minutes to hours following cerebral ischemia, so therapeutics aiming to inhibit this acute phase must be administered very shortly after the ischemic insult. Of relevance, this time course may match the first protection window occurring within minutes to hours after the induction of "rapid preconditioning". The time constraints of acute neuroprotection may be difficult to achieve in 
clinical practice, but testing a drug in both paradigms is important in estimating its neuroprotective capacity. We have therefore investigated neuroprotection by ALA and DHA in an in vivo transient model of global ischemia, in which neuronal death of CA1 hippocampal pyramidal cells is mainly driven by glutamate excitotoxicity (Pulsinelli and Brierley, 1979). Seven days after a $20 \mathrm{~min}$ global ischemia, only $15 \%$ of the CA1 neurons survived the ischemic challenge, while the injection of ALA (i.c.v., $10 \mu \mathrm{M}$ / $5 \mu \mathrm{L})$ within $30 \mathrm{~min}$ post-ischemia allowed $80 \%$ of the CA1 neurons to survive. ALA was also protective when administered intravenously (i.v., $500 \mathrm{nmol} / \mathrm{kg}$ ) 30 minutes before or after the ischemic challenge (figure $1 \mathrm{~A}$ ). However, in this model, EPA and DHA had less pronounced and reproducible protective effects (Lauritzen et al., 2000). We therefore decided to focus our investigation on the omega-3 precursor rather than on the long chain derivates. To clearly establish that ALA could inhibit glutamate excitotoxicity we tested its protective potential in an in vivo model of seizure induced by the administration of the glutamate analog kainic acid, and in an in vitro model of glutamate stress. In vivo, using the same dosage paradigms as for ischemia, ALA was neuroprotective when injected i.v. either 30 min before or after KA treatment (Lauritzen et al., 2000). In vitro, the same range of concentrations known to be protective both in vivo with i.c.v. injection and in vitro on granule cells, prevented hippocampal neuronal death triggered by the addition of an excitotoxic concentration of glutamate $(50 \mathrm{mM})$ for 24 hours (Blondeau et al., 2009). In all these models a saturated fatty acid, palmitic acid, failed to induce any beneficial effects, underlying the importance of the omega-3 polyunsaturated class of fatty acids.

ALA effect on the vascular tone and CBF was investigated. As previously discussed, the failures of therapeutics targeting only neurons and the hyperacute nature of glutamate releasedriven neuronal death in ischemia lead the scientific community to identify drugs that could also help restoring and/or preserving the cerebral blood flow (CBF). The in vivo and in vitro neuroprotective $10 \mu \mathrm{M}$ and $100 \mu \mathrm{M}$ concentrations of ALA induced an increase of the diameter of the basilar, but not of the carotid artery (Blondeau

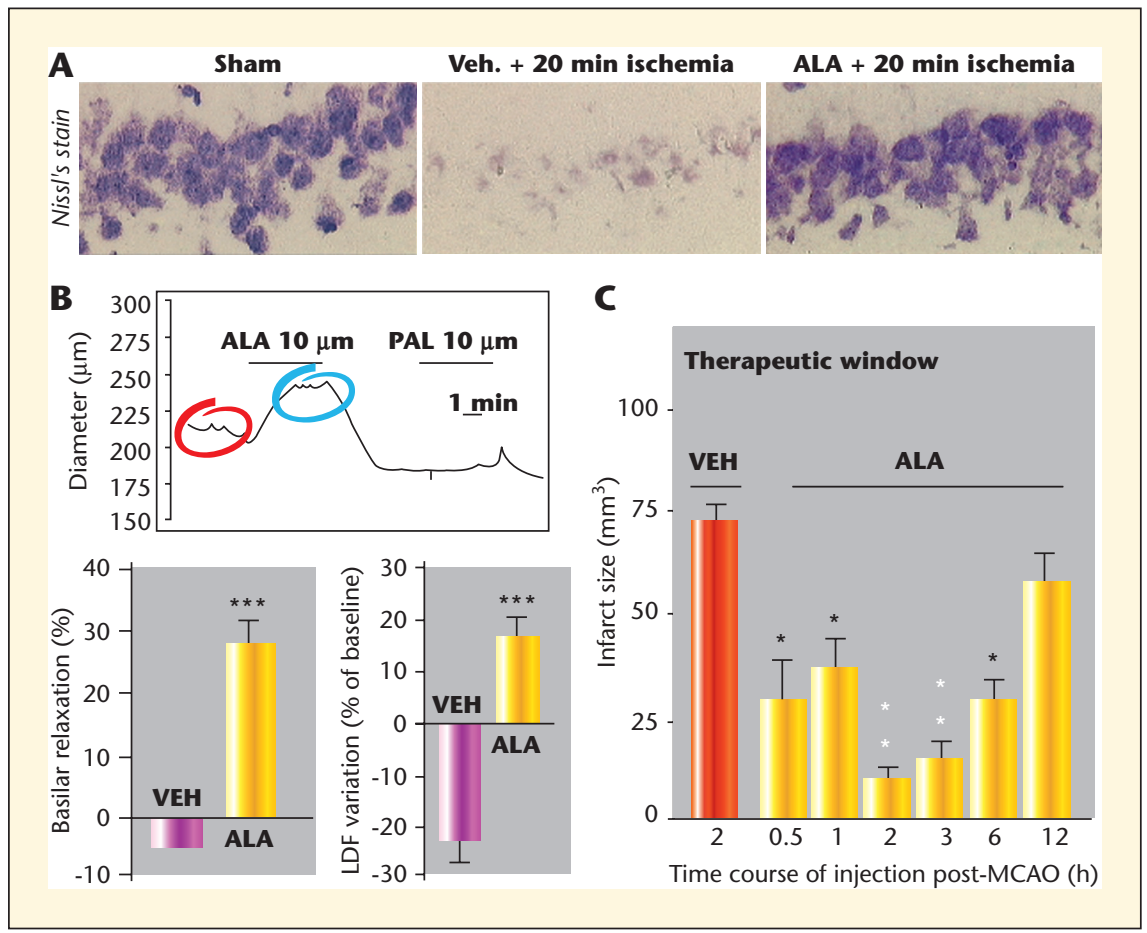

Figure 1. Omega-3 $\alpha$-linolenic acid decreases acute ischemic stroke damage. A) ALA intravenous injection (i.v., $500 \mathrm{nmol} / \mathrm{kg}$ ) 30 minutes before a ischemic challenge (20 min global ischemia) prevents glutamate excitotoxicity-driven CA1 hippocampal neuronal death. B) Left panel: ALA is vasoactive triggering a $30 \%$ release of the basilar artery diameter ex vivo. Right panel: In vivo a $20 \%$ increase of the CBF occurs within 30 min post ALA-injection (i.v. neuroprotective dose of $500 \mathrm{nmol} / \mathrm{kg}$ ). C) ALA significantly reduces the infarct volume when injected up to 6 hours after reperfusion post-MCAO. The maximal protection was achieved with a dose of $500 \mathrm{nmol} / \mathrm{kg}$ injected 2 hours post-ischemia and the window of successful intervention faded 12 hours after the onset of reperfusion.

et al., 2007). In both mice and rats (figure 1B) ALA acted as a vasoactive drug, leading to an approximately $30 \%$ increase of the diameter of the basilar artery ex vivo, accounting for a $20 \%$ increase of the in vivo CBF observed within 30 min post-injection (i.v. neuroprotective dose of $500 \mathrm{nmol} / \mathrm{kg}$ ). Since ALA did not dilate carotid arteries with elastic properties, the omega- 3 induced relaxation appears to be specific to cerebral resistance arteries such as those of the cerebral vascular bed, without affecting the systemic blood pressure. Therefore, both the increased resistance to glutamate hyperexcitability and vasodilation capacity of brain arteries that increases collateral flow and reduce CBF loss in the penumbra, after ALA injection, may potentially contribute to protection against ischemic stroke. Thus, we investigated the effect of $\alpha$ linolenic acid in the middle cerebral artery occlusion (MCAO) model, using the intraluminal suture technique ascribed to be the closest model to human stroke.
The compatibility of ALA post-treatment to the clinical setting was addressed by investigating two clinically relevant parameters. The window of intervention to efficiently reduce the infarct volume 24 hours post-MCAO was characterized, and a delivery protocol was designed to improve the long-term survival rate. ALA significantly reduced the infarct volume when injected up to $6 \mathrm{~h}$ after reperfusion onset (Heurteaux et al., 2006). Maximal protection was achieved using $500 \mathrm{nmol} / \mathrm{kg}$ injected $2 \mathrm{~h}$ post-ischemia, and the window of intervention faded by 12 hours after the onset of reperfusion (figure 1C). The ALA-induced neuroprotection correlated with a decrease in cytopathological features of cell injury, DNAfragmentation and pro-apoptotic Bax protein up-regulation. For all parameters used as a measure of the degree of protection, the natural omega-3 precursor efficiency was similar to riluzole, a chemical neuroprotectant currently in clinical use for amyotrophic lateral sclerosis. In contrast, the saturated 
palmitic fatty acid failed to reduce the infarct volume when injected 2 hours after reperfusion. Interestingly, a single ALA injection providing the best cerebral protection at 24 hours post-stroke had no beneficial effect on the longterm survival rate. To achieve a 3 -fold improvement in the survival rate at 10 days and one month post-ischemia, repeated injections were required (Blondeau et al., 2009, Heurteaux et al., 2006). This data suggested that the reduction of glutamate excitotoxicity was insufficient to reduce longterm mortality rates. Indeed, excitotoxicity suppression may instead only delay neuronal death, by failing to prevent later stages of cell death including apoptosis and associated inflammatory events, events known to cause progressive tissue damage hours to days or weeks later. Nevertheless, the beneficial effects caused by repeated injections spaced several days apart implied that ALA triggered other beneficial mechanisms. Preclinical data indicate that pharmacological therapies that enhance brain-repair processes substantially improve functional recovery when given during the later recovery phase of stroke. The concept underlying these restorative therapies is the pleiotropic targeting of many parenchymal cell types including neural stem cells, cerebral endothelial cells, astrocytes, oligodendrocytes, and neurons leads to enhancement of neurotrophic factor production, endogenous neurogenesis, angiogenesis, and synaptogenesis in ischemic brain tissue (Zhang and Chopp, 2009). These events collectively improve stroke outcome including neurological function.

To evaluate long-term benefits, the effect of subchronic ALA administration on neuronal plasticity was investigated. An increase in neurogenesis was observed, following three sequential injections performed using the same time course and dose that improved long-term survival rate post-stroke (Blondeau et al., 2009). Subchronic ALA treatment significantly increased the number of proliferating immature neurons, as identified by the colocalization of incorporated BrdU, a DNA synthesis marker, in dividing progenitor cells and DCX, a microtubule-associated protein specifically expressed in all migrating neuronal precursors. These immature neurons identified 3 days after the last injection survived and matured by 21 days after

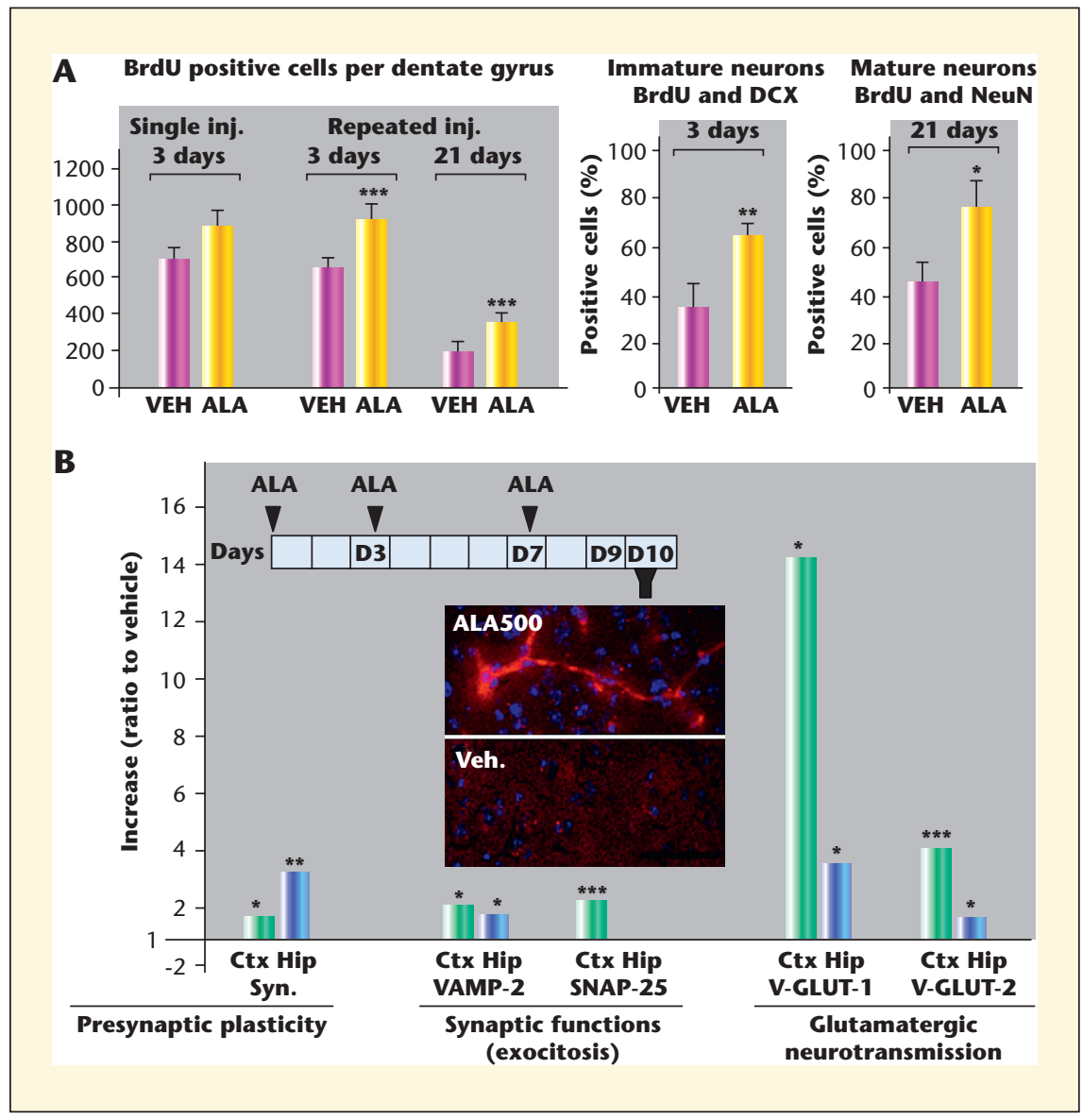

Figure 2. Omega-3 $\alpha$-linolenic acid increases neurogenesis and synaptogenesis. A) Three and 21 days after the subchronic ALA-treatment, cell proliferation is increased in the Dentate Gyrus (left panel). The proliferating cells are immature neurons identified 3 days after the last ALA injection and mature neurons 21 days later. B) ALA treatment increases the levels of different markers of synaptogenesis in cortex, hippocampus at Day 10. Highlighted in the box is immunostaining for SNAP-25, a synaptosome-associated exocytosis protein, in the cortex of ALA500-treated animals at Day 10 showing the increased in synaptogenesis by ALA treatment.

the last ALA injection (figure 2A), suggesting that the repeated ALA injections triggered the induction of neurogenesis. The upregulation of key proteins involved in synaptic functions, synaptophysin-1, VAMP-2, and SNAP25 as well as proteins supporting glutamatergic neurotransmission, VGLUT1 and V-GLUT2, also indicated that ALA subchronic treatment promoted synaptogenesis (figure $2 B$ ). These effects correlated with an increase in BDNF protein levels both in vivo, following subchronic ALA treatment and in vitro using neural stem cells and hippocampal cultures (Blondeau et al., 2009). Altogether these results imply a pleiotropic effect of ALA, which could target different brain cell types and combine acute neuroprotection,
CBF regulation and long-term repair/ compensatory plasticity to protect from stroke. This pleiotropism parallels brain preconditioning findings of combined neuroprotective, regenerative and antiinflammatory effects.

\section{Omega-3 $\alpha$-linolenic acid as a preconditioner in experimental model of cerebral ischemia and stroke}

As a proof a concept that ALA could be a "natural" preconditioner against cerebral ischemia, we tested whether ALA preconditioning could trigger the late phase of tolerance against two models of excitotoxicity-driving neuronal death 
by global ischemia and kainic acid injection. ALA injection (i.v., 500 $\mathrm{nmol} / \mathrm{kg}$ ) 3 days before $6 \mathrm{~min}$ global ischemia or kainic acid-induced epileptic seizure almost fully prevented the CA1 neurodegeneration (Blondeau et al., 2002b). The narrow temporal window of cerebral protection conferred by ALA preconditioning parallels findings observed using preconditioning by ischemia or epileptic activity. Similarities in signal transduction pathways activated with ischemia and other well-established chemical preconditioners, confirmed that ALA may be studied as a new natural preconditioner of the brain. For example, ALA preconditioning induced the neuroprotective HSP70 heat shock protein within a similar time frame and neuronal localization shared by ischemic, epileptic and adenosine and $\mathrm{K}_{\text {atp }}$ channel openers preconditioning (Blondeau et al., 2000; Blondeau et al., 2002b). In addition, preconditioning by ALA, ischemic, kainic acid-induced epileptiform activity rapidly increased the expression of the transcription factor nuclear factor- $\kappa \mathrm{B}$. NFкB is an ubiquitously expressed inducible regulator of a broad range of genes that play pivotal roles in cell death and survival pathways. The three different preconditionings increased NFkB DNA-binding activity and nuclear translocation of p65 and p50 subunits of NFkB in a similar manner and cellular compartmentalization (Blondeau et al., 2001). Pretreatment with the NFkB inhibitor diethyldithiocarbamate or kB-decoy DNA suppressed the increased DNA-binding activity and the nuclear translocation of NFkB, leading to the loss of the preconditioning-induced neuroprotection against an otherwise lethal ischemic or epileptic challenge. Thus the functions of the pathways established by ALA preconditionings are similar to those observed in the other preconditioning that are required for the development of brain tolerance. ALA preconditioning was also demonstrated in mice, since ALA subchronic treatment 3 days before a transient focal ischemia significantly reduced the infarct volume (Blondeau et al., 2009). The similarities in the narrow window of cerebral protection, as well as overlap in signal transduction pathways, activated by ischemia and other well-established chemical preconditioners, clearly demonstrates that ALA can trigger the ubiquitous pleiotropic protective signaling pathways important to brain preconditioning.

\section{Omega-3 $\alpha$-linolenic acid as preconditioner in the diet to prevent stroke- induced damage: from preconditioning toward nutraceutical}

The overall properties of ALA may fulfill criteria necessary to rank this omega-3 PUFA as a good neuroprotectant, or at least as a crucial molecule for brain resistance. ALA is a fatty acid qualified of "essential", because its synthesis is not performed by the human organism and must therefore be provided by diet. This could explain in part why the severe deficiency in omega-3 intake that has been reported in numerous epidemiologic studies may weaken the brain, representing an important risk factor in the development and/or deterioration of certain neuropathologies and neurovascular diseases, like stroke. In the context of stroke treatment, the rationale of supplementation with a non-ischemic preconditioner like ALA has seemed promising to investigate, especially since no detrimental effects of ALA have been documented as far as we are aware. An unobtrusive method of increasing ALA intake would be to increase levels in the daily diet. We addressed this important hypothesis by investigating whether ALA supplementation achieved using a diet enriched in rapeseed oil, a rich source of ALA as the only source of lipids, decreased infarct volumes in stroked mice. A diet enriched with ALA (0,75\% by weight) significantly reduced mortality rate, infarct size induced by $60 \mathrm{~min}$ of middle carotid artery occlusion and increased the probability of spontaneous reperfusion in the post-ischemic period (Nguemeni et al., 2010). Figure 3 shows that the ALA-enriched diet decreased the total infarct volume by 35 to $45 \%$ (total infarct, cortical infarct). The reduction of the focal ischemic lesion was similar in rats supplemented with high dietary levels of long-chain omega-3 (1.75\% by weight) over 6 weeks (Relton et al., 1993). This result implies that lower levels of supplementation may be

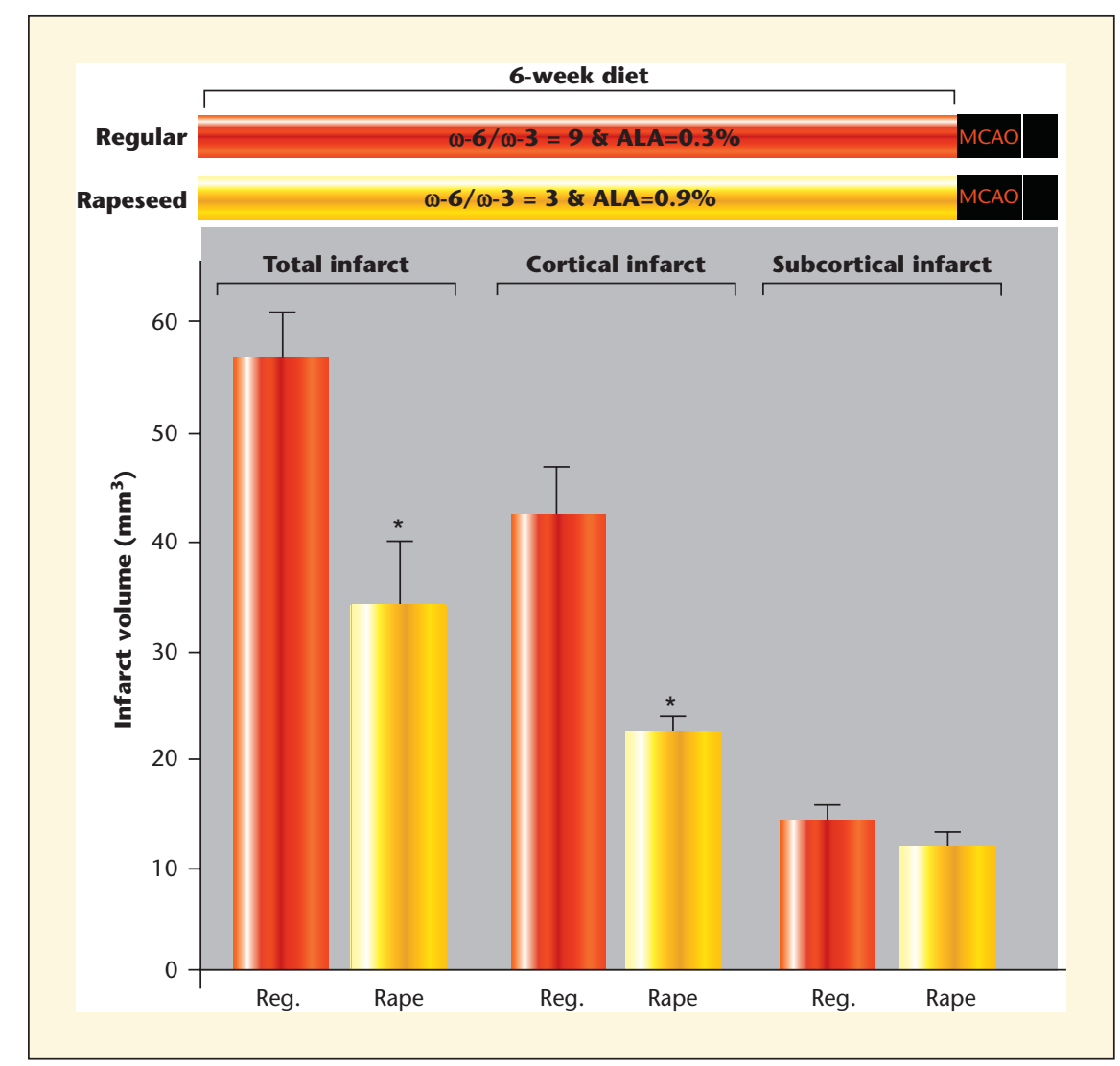

Figure 3. ALA-enriched diet decreases the infarct volume induced by 60 min MCAO. The upper panel summarizes the 6-week diet protocol and the ALA content in the diet. The bottom panel shows that the cerebroprotection occurs mainly at the cortical level. 
required when using precursors compared to long-chain derivatives. Since the neuronal protection obtained with supplementation achieved force-feeding fish oil rich in long-chain omega-3 is controversial (Bas et al., 2007; Ozen et al., 2008), ALA provided by vegetable rapeseed oil should be considered as an interesting alternative to longchain omega-3 derivates from fish oil. Finally, a drastic reduction of lipid peroxidation levels was also observed in the ischemic brain of animals fed the ALA enriched diet, suggesting that beneficial effects of ALA have not yet been exhaustively categorized (Nguemeni et al., 2010).

\section{Conclusion}

To conclude, this review supports $\alpha$ linolenic acid as an omega-3 precursor exhibiting interesting potential therapeutic value against stroke. Moreover, a new perspective in the preconditioning field is offered. Specifically, a novel approach to precondition the brain against ischemia may be to implement existing or novel nutraceuticals. Indeed, one could view nutraceuticals as "natural preconditioners" which target the brain to its increase resistance against devastating insults such as stroke. Ultimately, the future of preconditioning largely depends upon successful translation of important findings to daily life and the clinical arena. Development of strategies achieved using functional food may represent an important avenue to achieve this goal.

Acknowledgments. Ideas discussed here are based in part on the author's presentation at the 2011 Journées CHEVREUL - Lipids and Brain 2 The French Society for the Study of Lipids in Paris. I am grateful to GLN, ONIDOL, the "Fondation de la Recherche Médicale" and CNRS for their support. I wish to thank Dr Joseph Tauskela for his critical reading of the manuscript. I am also grateful to Pr Bernadette Delplanque and Dr Catherine Heurteaux for many helpful discussions. Finally, I thank all our past and present team members who have contributed to the data discussed in the review.

\section{REFERENCES}

Allen CL, Bayraktutan U. Risk factors for ischaemic stroke Int J Stroke 2008; 3: 105-16.
Bas O, Songur A, Sahin O, et al. The protective effect of fish n-3 fatty acids on cerebral ischemia in rat hippocampus Neurochem Int 2007; 50: 548-54.

Blondeau N, Lauritzen I, Widmann C, Lazdunski M, Heurteaux C. A potent protective role of lysophospholipids against global cerebral ischemia and glutamate excitotoxicity in neuronal cultures. I Cereb Blood Flow Metab 2002a; 22: 821-34.

Blondeau N, Nguemeni C, Debruyne DN, et al. Subchronic alpha-linolenic acid treatment enhances brain plasticity and exerts an antidepressant effect: a versatile potential therapy for stroke. Neuropsychopharmacology 2009; 34: 2548-59.

Blondeau N, Petrault O, Manta S, et al. Polyunsaturated fatty acids are cerebral vasodilators via the TREK-1 potassium channel. Circ Res 2007; 101: 176-84.

Blondeau N, Plamondon H, Richelme C, Heurteaux C, Lazdunski M. K(ATP) channel openers, adenosine agonists and epileptic preconditioning are stress signals inducing hippocampal neuroprotection. Neuroscience 2000; 100: 465-74.

Blondeau N, Widmann C, Lazdunski M, Heurteaux C. Activation of the nuclear factor-kappaB is a key event in brain tolerance. / Neurosci 2001; 21: 4668-77.

Blondeau N, Widmann C, Lazdunski M, Heurteaux C. Polyunsaturated fatty acids induce ischemic and epileptic tolerance. Neuroscience 2002b; 109: 231-41.

Dauchet L, Amouyel P, Dallongeville J. Fruit and vegetable consumption and risk of stroke: a meta-analysis of cohort studies. Neurology 2005; 65: 1193-7.

de Goede J, Verschuren WM, Boer JM, Kromhout D, Geleijnse JM. Alpha-linolenic Acid intake and 10-year incidence of coronary heart disease and stroke in 20,000 middle-aged men and women in the Netherlands. PLoS One 2011; 6: e17967.

Dirnagl U, Simon RP, Hallenbeck JM. Ischemic tolerance and endogenous neuroprotection. Trends Neurosci 2003; 26: 248-54.

Endres $\mathrm{M}$, Dirnagl U. Ischemia and stroke. Adv Exp Med Biol 2002; 513: 455-73.

Fink $M$, Lesage $F$, Duprat $F$, et al. A neuronal two $\mathrm{P}$ domain $\mathrm{K}+$ channel stimulated by arachidonic acid and polyunsaturated fatty acids. EMBO / 1998; 17: 3297-308.

Gidday JM. Cerebral preconditioning and ischaemic tolerance. Nat Rev Neurosci 2006; 7: 437-48.

Heurteaux C, Laigle C, Blondeau N, Jarretou G, Lazdunski M. Alpha-linolenic acid and riluzole treatment confer cerebral protection and improve survival after focal brain ischemia. Neuroscience 2006; 137: 241-51. ladecola C. Neurovascular regulation in the normal brain and in Alzheimer's disease. Nat Rev Neurosci 2004; 5: 347-60.

Jonas S, Ayigari V, Viera D, Waterman P. Neuroprotection against cerebral ischemia. A review of animal studies and correlation with human trial results. Ann N Y Acad Sci 1999; 890: 2-3.

Kirino T. Ischemic tolerance. / Cereb Blood Flow Metab 2002; 22: 1283-96.

Kitagawa K, Matsumoto M, Tagaya M, et al. 'Ischemic tolerance' phenomenon found in the brain. Brain Res 1990; 528: 21-4.

Lauritzen I, Blondeau N, Heurteaux C, Widmann C, Romey G, Lazdunski M. Polyunsaturated fatty acids are potent neuroprotectors. EMBO / 2000; 19: 1784-93.

Lloyd-Jones D, Adams RJ, Brown TM, et al. Heart disease and stroke statistics-2010 update: a report from the American Heart Association. Circulation 2010; 121: e46e215.

Lo EH. Experimental models, neurovascular mechanisms and translational issues in stroke research. Br J Pharmacol 2008; 153 (Suppl. 1): S396-405.

Lo EH, Rosenberg GA. The neurovascular unit in health and disease: introduction. Stroke 2009; 40: S2-3.

Minnerup J, Schabitz WR. Multifunctional actions of approved and candidate stroke drugs. Neurotherapeutics 2009; 6: 43-52.

Moskowitz MA, Lo EH, ladecola C. The science of stroke: mechanisms in search of treatments. Neuron 2010; 67: 181-98.

Nguemeni C, Delplanque B, Rovere C, et al. Dietary supplementation of alpha-linolenic acid in an enriched rapeseed oil diet protects from stroke. Pharmacol Res 2010, 61; 226-33.

O'Duffy AE, Bordelon YM, McLaughlin B. Killer proteases and little strokes-how the things that do not kill you make you stronger. J Cereb Blood Flow Metab 2007; 27: 655-68.

Obrenovitch TP. Molecular physiology of preconditioning-induced brain tolerance to ischemia. Physiol Rev 2008; 88: 211-47.

Ozen OA, Cosar M, Sahin O, et al. The protective effect of fish n-3 fatty acids on cerebral ischemia in rat prefrontal cortex. Neurol Sci 2008; 29: 147-52.

Plamondon $\mathrm{H}$, Blondeau $\mathrm{N}$, Heurteaux C, Lazdunski M. Mutually protective actions of kainic acid epileptic preconditioning and sublethal global ischemia on hippocampal neuronal death: involvement of adenosine A1 receptors and K(ATP) channels. I Cereb Blood Flow Metab 1999; 19: 1296-308.

Pulsinelli WA, Brierley JB. A new model of bilateral hemispheric ischemia in the unanesthetized rat. Stroke 1979; 10: 267-72. 
Relton JK, Strijbos PJ, Cooper AL, Rothwell NJ. Dietary N-3 fatty acids inhibit ischaemic and excitotoxic brain damage in the rat. Brain Res Bull 1993; 32: 223-6.

Riediger ND, Othman RA, Suh M, Moghadasian $\mathrm{MH}$. A systemic review of the roles of $\mathrm{n}-3$ fatty acids in health and disease. J Am Diet Assoc 2009; 109: 668-79.

Rosamond W, Flegal K, Furie K, et al. Heart disease and stroke statistics-2008 update: a report from the American Heart Association Statistics Committee and Stroke Statistics
Subcommittee. Circulation 2008; 117: e25146.

Simopoulos AP. The importance of the omega-6/omega-3 fatty acid ratio in cardiovascular disease and other chronic diseases. Exp Biol Med (Maywood) 2008; 233: 674-88.

Tauskela JS, Blondeau N. 2009 In: Ischemic Tolerance of the Brain, Research Signpost. Schaller, B], Ed.; Kerala, India : Research Signpost, 2009: 85-135.

Weih M, Kallenberg $\mathrm{K}$, Bergk $\mathrm{A}$, et al. Attenuated stroke severity after prodromal
TIA: a role for ischemic tolerance in the brain? Stroke 1999; 30: 1851-4.

Zaleska MM, Mercado ML, Chavez J, Feuerstein GZ, Pangalos MN, Wood A. The development of stroke therapeutics: promising mechanisms and translational challenges. Neuropharmacology 2009; 56: 329-41.

Zhang ZG, Chopp M. Neurorestorative therapies for stroke: underlying mechanisms and translation to the clinic. Lancet Neurol 2009; 8: 491-500. 